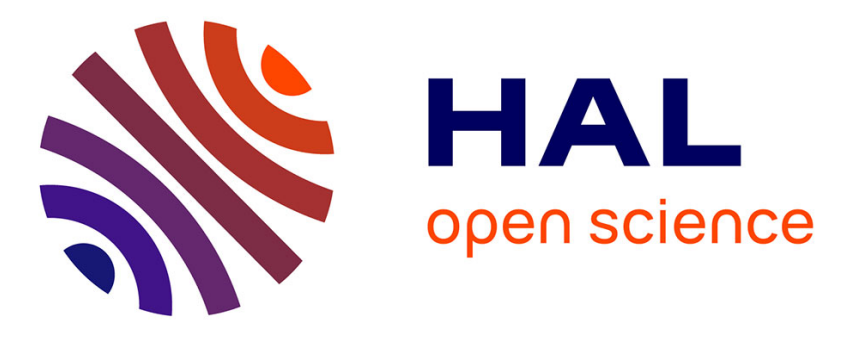

\title{
Une modélisation de l'identité-logement pour une éducation de la personne âgée au risque de chute à domicile
}

Frédérique Trévidy, Jean-François d’Ivernois, Jean-Jacques Mourad, Guillaume Brugidou, Rémi Gagnayre

\section{To cite this version:}

Frédérique Trévidy, Jean-François d'Ivernois, Jean-Jacques Mourad, Guillaume Brugidou, Rémi Gagnayre. Une modélisation de l'identité-logement pour une éducation de la personne âgée au risque de chute à domicile. Éducation thérapeutique du patient / Therapeutic patient education , 2015, 7 (1), pp.10102. 10.1051/tpe/2015001 . hal-01568958

\section{HAL Id: hal-01568958 \\ https://sorbonne-paris-nord.hal.science/hal-01568958}

Submitted on 26 Jul 2017

HAL is a multi-disciplinary open access archive for the deposit and dissemination of scientific research documents, whether they are published or not. The documents may come from teaching and research institutions in France or abroad, or from public or private research centers.
L'archive ouverte pluridisciplinaire HAL, est destinée au dépôt et à la diffusion de documents scientifiques de niveau recherche, publiés ou non, émanant des établissements d'enseignement et de recherche français ou étrangers, des laboratoires publics ou privés. 


\title{
Article original/Original article
}

\section{Une modélisation de l'identité-logement pour une éducation de la personne âgée au risque de chute à domicile}

\author{
Frédérique Trévidy ${ }^{1,2 \star}$, Jean-François d'Ivernois ${ }^{1}$, Jean-Jacques Mourad ${ }^{1,3}$, Guillaume Brugidou ${ }^{2}$, \\ Rémi Gagnayre $^{1}$ \\ 1 Laboratoire Éducations et Pratiques de Santé, EA 3412, Université Paris 13, Sorbonne Paris Cité, France \\ 2 ALFI, Groupe Arcade, 59 rue de Provence, 75009 Paris, France \\ 3 Centre Hospitalier Universitaire Avicenne, France
}

(Reçu le 21 octobre 2014, accepté le 16 février 2015 )

\begin{abstract}
Résumé - Introduction : Les préconisations professionnelles visant à réduire les risques domiciliaires de chute sont moyennement suivies par les personnes âgées. Nous présumons l'existence d'une identité-logement (IL) à l'origine du rapport complexe entre l'individu et son lieu de vie. Objectifs : Cette recherche qualitative vise dans un premier temps à modéliser les processus d'apprentissage constituant l'IL pour envisager, dans un second temps, une éducation thérapeutique du patient (ETP) chuteur lui permettant de participer pleinement à l'aménagement de son domicile. Méthode : Notre méthode emprunte de la théorisation ancrée. Dix entretiens semi directifs ont été menés auprès de seniors chuteurs avec l'utilisation d'une carte mentale du logement (CML). Résultats : Le modèle de l'IL comprend trois propriétés : a. Le sentiment de continuité temporelle, cœur de l'IL, se compose des routines et connaissances de l'individu dans son cycle du présent, liées à ses souvenirs et projections futures. b. Le sentiment d'unité et de cohérence contient le sentiment de continuité, et impulse chez l'habitant trois types de stratégies. c. Les stratégies d'intégration et d'adaptation déclenchent des processus d'apprentissage et font évoluer l'IL alors que les stratégies de défense la protègent mais limitent l'apprentissage. Discussion : Dispensé au domicile et centré sur l'habitant, le programme d'ETP comprend : un diagnostic éducatif - une éducation à l'aménagement et au risque de chute - un accompagnement de la personne âgée pendant les travaux. La CML serait utilisée pour caractériser l'évolution de l'IL. Conclusion : Cette éducation spécifique tenant compte de l'IL pourrait intégrer à terme des programmes d'ETP existants sur la prévention de la chute (ex : PAERPA).
\end{abstract}

Mots clés : identité-logement / chute / personnes âgées / modélisation / éducation thérapeutique

\begin{abstract}
A model of home-identity for an education of elderly on risk of falling in housing. Introduction: The professional recommendations to reduce the risk of falling in housing are moderately followed by the elderly. We assume the existence of a "home-identity" (HI) behind the complex relationship between the individual and his habitation. Objectives: This qualitative research aims initially to model the learning process for constituting the HI consider, at a later stage, a therapeutic patient education (TPE), which would allow elderly fallers to fully participate in the modification of their home. Method: Our method is based on the grounded theory. Ten semi-structured interviews were conducted with the older fallers with the use of a mental map of the home (MMH). Results: The model of HI consists of three properties: a. The sense of temporal continuity, heart of HI, consists of routines and knowledge of the individual in the present cycle, linked to memories and future projections. b. The sense of unity and coherence contains the sense of continuity, and impulses three kinds of strategies at the inhabitant. c. Integration and adaptation strategies trigger the learning process and change the HI. The defense strategies protect the HI but limit the learning. Discussion: provided at home and centered on the inhabitant, the TPE program would include: an educational diagnosis - an education on the housing's modifications and the risk of falling - an accompanying of the elderly during work. The $\mathrm{MMH}$ is used to characterize the evolution of the HI. Conclusion: This specific education into account the HI could eventually integrate the existing TPE programs on the prevention of falls (ex : PAERPA).
\end{abstract}

Key words: home-identity / falls / elderly / modeling / therapeutic patient education

Abréviations : M1 : madame L. ; F1 : fille de madame L; M2 : madame R.; F2 : fils de madame R; M3 : madame C; M4 : madame S. ; M5 : monsieur Y ; M6 : monsieur G. ; M7 : madame H.; M9 : madame A ; M10 : madame D ; A 10 : petite fille de madame $\mathrm{D} ; \mathrm{C}$ : chercheure.

^ Correspondance : Frédérique Trévidy, fredtrevidy@aliceadsl.fr 


\section{Introduction}

Selon les revues internationales, environ $30 \%$ des personnes de plus de 65 ans chutent chaque année [1]. Ces chutes proviennent principalement à domicile $(78 \%)$ [2] et peuvent engendrer un syndrome post-chute [3] lié à la peur de chuter, provoquant une restriction des activités, une perte d'autonomie, un déconditionnement de capacités physiques de ces personnes [4] et une augmentation de la récidive de chute [5].

En France, on compte 81,3 décès par chute sur 100000 personnes de plus de 65 ans [6]. Au vu des conséquences tant physiologiques que sociales, la prévention de la chute est devenue un enjeu de santé publique. Plusieurs facteurs de risque sont identifiés $[3,7-11]$ tels que des facteurs sociodémographiques (âge avancé, sexe féminin, etc.), des facteurs liés à la personne (pathologies aiguës ou chroniques, troubles de la marche, etc.), à son comportement (consommation d'alcool, activités risquées, port de lunettes inappropriées) ou à l'environnement (dangers du domicile, risques extérieurs, etc.). Les recommandations professionnelles préconisent d'agir auprès des personnes présentant un risque élevé de chute par le biais d'interventions multifactorielles personnalisées [3,7-9] comprenant plusieurs actions préventives dont la réduction des dangers du domicile. Bien que la sécurisation du lieu de vie est reconnue comme une action efficace [12], moins de la moitié des aménagements préconisés par les professionnels sont suivis par les personnes âgées [3]. Le coût des travaux peut représenter un frein au changement [13] mais cette réalité ne doit pas occulter une autre dimension, davantage subjective, qui expliquerait cette réticence des personnes âgées à vouloir modifier leur intérieur pour sécuriser leur domicile. C'est ce que mettent en évidence Clément et al. à travers leur analyse de la littérature sur le vieillissement de la population. Ils observent en effet une certaine aversion des anciens vis-à-vis des objets marqueurs de handicap : «les objets marqueurs de handicap sont rarement retenus ou vite abandonnés au profit d'objets ordinaires achetés ou bricolés » ([14], p. 67). Au-delà des aménagements, c'est la perception même du risque de chute par les personnes âgées au domicile qui est interrogée par d'autres chercheurs. Ainsi, les résultats du programme SADEER visant à évaluer l'impact de l'intervention au domicile des ergothérapeutes dans la cadre de la prévention des désadaptations habitat-habitant mettent en lumière la difficulté des seniors à prendre conscience des risques malgré les difficultés rencontrées au quotidien [15]. Cette dimension subjective du lien entre la personne âgée et son logement questionne le rapport identitaire que cette dernière peut établir avec son lieu de vie. Dans la maison, les habitudes quotidiennes, l'histoire portée par les objets, l'attachement aux meubles peuvent former un univers d'autant plus impérieux que l'habitant vieillit avec eux. Au fil du temps, la personne a pu poser sur son intérieur des significations particulières et développé un lien singulier avec son logement que nous nommons «identité-logement » [16].

Pour prévenir la chute, l'INPES recommande de développer une approche éducative permettant de favoriser une dynamique de changement chez l'individu en tenant compte de ses croyances, représentations et compétences [3].
L'expérimentation PAERPA (Personnes âgées en risque de perte d'autonomie) menée par la HAS préconise une éducation thérapeutique du patient dans le cadre des programmes multifactoriels destinés aux personnes à haut risque de chute [17]. Cette éducation pourrait s'enrichir si elle intégrait une intervention spécifique sur le logement respectueuse de l'identitélogement de la personne âgée.

\section{Cadre théorique et objectifs de la recherche}

Le logement n'est pas neutre pour l'habitant. Djaoui le qualifie d'espace psychique considéré comme «une zone intermédiaire entre l'espace intérieur du sujet et la réalité extérieure » ([18], p. 11). Le domicile relève de la subjectivité la plus absolue de chaque habitant en accueillant ses valeurs, ses sentiments, ses représentations [18]. Ennuyer évoque le domicile comme un lieu "d'identité psychique» ([19], p. 65). D'autres auteurs le conçoivent comme un espace onirique [20], lequel peut devenir « un palais de mémoire» pour les personnes âgées [21]. La maison prend la forme d'une construction symbolique, se vivant comme le corps sur des modes d'appropriation et de possession [22]. Par ailleurs, le logement accueille les usages de l'habitant, les fonctions affectées à chaque pièce - davantage marquées chez les plus âgés - [23], les «modes de vie d'habiter» déterminants pour l'utilisation des objets [24]. Ces deux dimensions à la fois subjective et d'usage du logement interrogent la relation développée par la personne avec son lieu de vie. Nous amènerons l'idée de l'existence possible d'une identité-logement [16].

Les choix théoriques pour élaborer le concept d'identitélogement reposent sur la prise en compte de la relation entre le sujet et son environnement d'une part, et sur la conception de ce lien comme source d'apprentissage pour l'individu, d'autre part. Selon l'approche constructiviste, l'activité du sujet lui permet de construire ses connaissances et d'établir un équilibre entre son intelligence et l'univers [25]. Grâce à sa structure cognitive, l'individu construit sa propre réalité du monde au sein duquel de nouvelles informations viendront se confronter à ses connaissances antérieures, rendant ainsi possible leur évolution. L'environnement est donc à la fois le produit et la source de l'apprentissage du sujet.

La psychologie environnementale permet d'étudier «l'individu dans son contexte physique et social en vue de dégager la logique des interrelations entre l'individu et son environnement en mettant en évidence, les perceptions, attitudes, évaluations et représentations environnementales d'une part, et les comportements et conduites environnementales qui les accompagnent, d'autre part » ([26], p. 19).

L'approche constructiviste et la psychologie environnementale constituent les deux volets respectivement théorique et disciplinaire, forgeant le cadre conceptuel de l'identitélogement.

En premier lieu, l'identité-logement est envisagée comme une identité spatiale - concept issu de la psychologie environnementale - au sens défini par Proshansky [27]. Cette 
identité est fondée sur la relation entre l'individu et son environnement et composée "d'un ensemble complexe d'idées conscientes et inconscientes, de croyances, de préférences, de sentiments de valeurs, de buts, de tendances et d'aptitudes comportementales liées à l'environnement» ([26], p. 80). Le modèle écologique de Bronfenbrenner [28] sert d'appui dans la mesure où il offre une approche systémique plaçant le sujet au centre d'un système environnemental. En adaptant ce modèle au concept de l'identité-logement, la personne âgée se situerait au centre d'un écosystème composé de quatre niveaux sociospatiaux concentriques allant du microsystème (logement/habitants), passant par le mésosystème (quartier/voisins, gardiens d'immeuble, espaces partagés/famille élargie, etc.), puis l'exosystème (environnements collectifs/collectivités locales et territoriales, etc.) jusqu'au macrosystème (société/valeurs sociales, pouvoir politique, médias). Cet environnement sociospatial est traversé par le chronosystème représentant la temporalité de l'individu. Chacune des sphères aurait une influence sur le développement de l'identité.

En second lieu, cette identité-logement est conçue selon une perspective constructiviste. Mucchielli définit l'identité comme une identité construite et située : «L'identité-située d'un acteur social tient compte du fait que le phénomène identitaire s'inscrit toujours dans une expérience de l'existence» ([29], p. 37). Selon cet auteur, le sentiment d'identité se composerait de plusieurs éléments tels que le sentiment d'appartenance, le sentiment d'unité et de cohérence, le sentiment de continuité temporelle, le sentiment de différence, le sentiment de valeur, le sentiment d'autonomie, le sentiment de confiance et enfin le sentiment d'existence [29]. Deux sentiments s'avèrent pertinents pour fonder le concept de l'identitélogement :

- le sentiment de continuité temporelle (SCT) permettant à l'individu d'établir des liens entre ses souvenirs, son présent et ses représentations futures. Grâce à ce sentiment, la personne construit son identité dans un environnement temporel (chronosystème);

- le sentiment d'unité et de cohérence (SUC) permettant à l'individu de rassembler toutes les identités bâties lors d'interactions avec d'autres acteurs, en une seule unité, assurant ainsi la cohérence de son être. Ce sentiment donne la possibilité à l'habitant de construire son identité dans un environnement sociospatial composé de plusieurs niveaux d'interrelations entre individus.

Ainsi, l'identité-logement, composée de deux sentiments sensibles au contexte social spatial et temporel, n'est pas définie à priori mais résulte d'une construction de l'individu selon la situation dans laquelle il se trouve dans l'interrelation développée avec son environnement. L'identité-logement serait évolutive grâce à des processus d'apprentissage lui étant liés.

Les objectifs de la recherche viseront dans un premier temps, à définir le concept de l'identité-logement à partir de ses deux sentiments constitutifs (SUC et SCT). Il s'agira ensuite de modéliser ce concept pour faire apparaître les processus d'apprentissage de l'individu, inhérents à cette identité-logement. La finalité consistera à proposer une intervention éducative qui s'appuierait sur ce modèle d'identitélogement. Elle pourrait donner les moyens à la personne âgée de plus de 65 ans ayant déjà chuté, d'apprendre à modifier son domicile pour le rendre plus sûr, en partant des éléments constitutifs de son identité.

\section{Méthodes}

\section{1 Échantillonnage par choix raisonné et théorique}

L'échantillon a été composé par choix raisonné en sélectionnant des personnes âgées de 65 ans et plus, ayant déjà chuté et vivant à domicile. Les personnes âgées vivant en institution ou sans antécédent de chute ont été exclues de l'étude. Tenant compte de ces critères d'inclusion, un échantillonnage théorique a été réalisé grâce à des variations au sein de l'échantillon initial, utiles aux besoins de l'étude (locataire de logement social/propriétaire/locataire de logement privé; appartement/pavillon; couple/isolé; réseau de ressources professionnelles/aidants familiaux) (Tab. I). Le repérage des personnes respectant les critères d'inclusion a été opéré par un organisme HLM francilien, un centre local d'information et de coordination de Seine Saint-Denis et une association caritative parisienne. Les sujets âgés ayant donné leur accord pour être mis en relation furent contactés téléphoniquement par le chercheur pour une présentation des objectifs de l'étude. Au total, dix ménages âgés de 66 à 95 ans - composés d'une personne seule ou d'un couple vivant dans le logement - donnèrent leur accord oral pour participer à cette recherche. Les interviews ont toutes été réalisées au domicile. Les personnes furent interrogées sur leur perception du risque de chute au domicile, leur utilisation des pièces, leurs trajets dans le logement et leurs souhaits d'aménagement pour sécuriser leur lieu de vie. Cinq ménages interrogés ont souhaité inviter un membre de l'entourage à participer à l'entretien.

\subsection{Recueil de données à l'aide d'une carte mentale du logement}

L'entretien semi-directif fut utilisé pour recueillir les données auprès des sujets, au moyen d'un guide d'entretien accompagné d'une Carte Mentale du Logement (CML) (Fig. 1), outil spécialement élaboré pour cette étude. La psychologie environnementale recommande de croiser plusieurs techniques de recueil pour illustrer le lien entre l'individu et son environnement. Les cartes mentales peuvent être utilisées dans le but de faire ressortir les représentations spatiales et le comportement «in situ» de l'individu [26].

Dessinée en début d'entretien par l'habitant ou par un proche, la CML permet de donner une illustration du logement qui servira de support au déroulé de l'interview. Les questions composant le guide se répartissent en trois domaines : « la perception du risque de chute en lien avec le logement», «la perception de l'aménagement du logement», «l'identification de la personne». Le sujet complète la carte en illustrant ses réponses au guide d'entretien, à l'aide de symboles couleur : en 
Tableau I. Profil des sujets inclus dans l'étude. - Profile of the subjects included in the study.

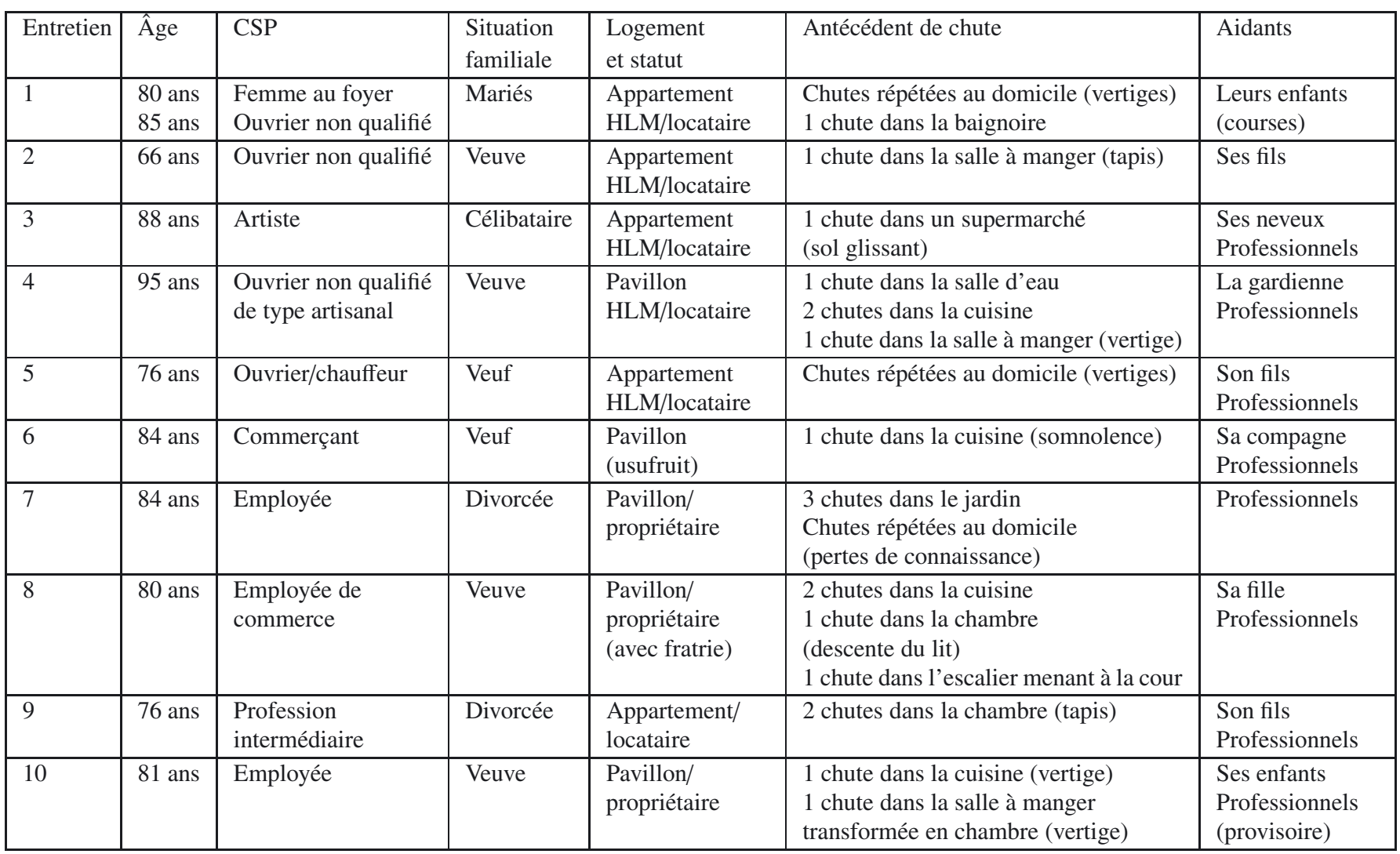

premier, les endroits appréciés et utilisés dans le logement, en second les lieux de chute (points noirs), les trajets effectués couramment (trait bleu) et ceux lui paraissant dangereux (trait rouge), les endroits jugés risqués (point rouge), et en troisième, les perceptions d'aménagement du logement nécessitant une adaptation du logement pour réduire ce risque (trait vert).

Le guide d'entretien et la CML ont été modifiés au cours de la recherche pour trois raisons :

- pour améliorer la qualité d'utilisation des outils, l'ordre des questions a été remanié (meilleure fluidité de l'entretien) et la carte mentale a été enrichie d'un visuel avec des symboles de couleur;

- pour élargir l'échantillon initial (échantillonnage théorique), des reformulations ont été appliquées au préambule du guide d'entretien ou à certaines questions visant à considérer de nouveaux espaces tels que des cours intérieures ou des jardins pour les pavillons ;

- pour vérifier les résultats obtenus au cours de la recherche, des questions ont été ajoutées sur le guide des entretiens 9 et 10 .

\subsection{La théorisation ancrée comme méthode d'analyse}

Dans le but d'explorer le phénomène social correspondant aux processus d'apprentissage internes à l'identité-logement, nous avons utilisé la théorisation ancrée [30], méthode d'analyse qualitative issue de la théorie ancrée [31], dont l'objectif n'est pas d'aboutir à un résultat (la théorie) mais de décrire le processus de théorisation. Cette méthode nous a servi de guide pour cheminer vers une modélisation de l'identité-logement en construisant des catégories et en les articulant de façon dynamique, à partir d'une codification des entretiens réalisés. Pour permettre une simultanéité entre le recueil et l'analyse des données, nous avons entrepris quatre phases de collecte empirique. Les résultats intermédiaires obtenus à la fin de chaque phase nous permettaient d'une part d'orienter la phase de recueil suivante en faisant varier l'échantillonnage et le guide d'entretien, et d'autre part, de poursuivre notre analyse à partir d'éléments théoriques de plus en plus élaborés : codes d'apprentissage, catégories composant les sentiments d'identité, mise en lien des catégories, modèle de l'identité-logement. Nos phases de recueil et d'analyse de données ont suivi les étapes de la théorisation ancrée proposées par Paillé, jusqu'au processus de modélisation [30]. La première étape a consisté à élaborer une codification des verbatim issus des trois premiers entretiens. Des codes thématiques sont apparus. Si certains thèmes résultaient directement des questions du guide d'entretien (chute, risque de chute, aménagement du logement, endroits, objets), d'autres émergeaient de l'expression des sujets interviewés (mésosystème, exosystème, soi et le logement, soi). Ces codes thématiques ont été analysés avec une seconde lecture orientée sur l'apprentissage, qui a donné naissance aux codes d'apprentissage, lesquels ont été testés et modifiés suite aux entretiens 4 et 5. Au total, 27 codes d'apprentissage ont été 


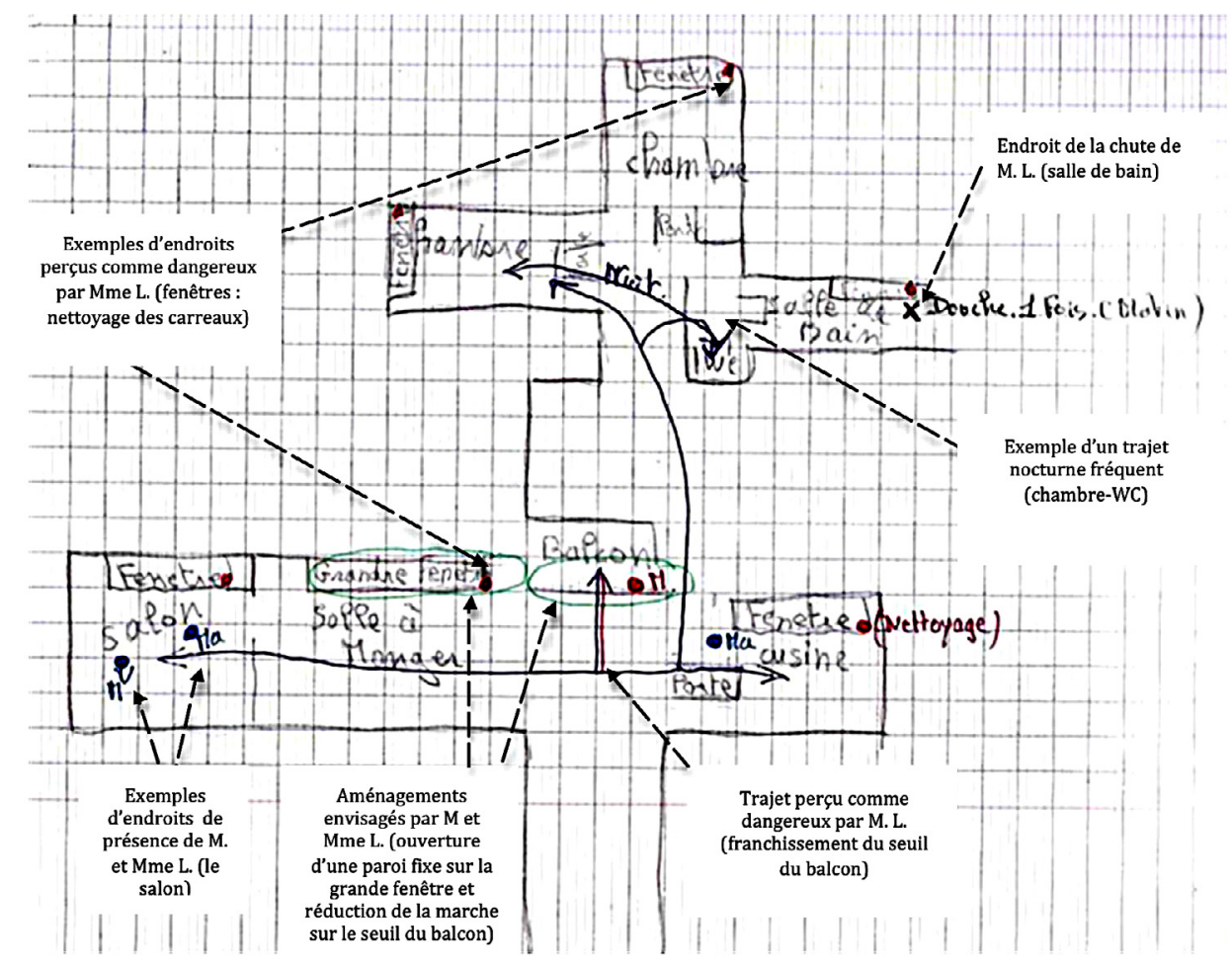

\begin{tabular}{|l|c|c|c|c|c|c|c|}
\hline Descriptif & $\begin{array}{c}\text { Endroits } \\
\text { des } \\
\text { chutes }\end{array}$ & $\begin{array}{c}\text { Endroits } \\
\text { de } \\
\text { présence }\end{array}$ & $\begin{array}{c}\text { Endroits } \\
\text { perçus } \\
\text { comme } \\
\text { dangereux }\end{array}$ & $\begin{array}{c}\text { Trajets } \\
\text { fréquents }\end{array}$ & $\begin{array}{c}\text { Trajets } \\
\text { perçus } \\
\text { comme } \\
\text { dangereux }\end{array}$ & $\begin{array}{c}\text { Aménagements } \\
\text { envisagés pour } \\
\text { réduire le risque }\end{array}$ & Annotations \\
\hline Symbole & Noir & Bleu & Rouge & Bleu & Rouge & Vert & $\begin{array}{c}\text { «Ma»: Madame } \\
\text { "M } \text { »: } \\
\text { Monsieur }\end{array}$ \\
\hline
\end{tabular}

Figure 1. La carte mentale du logement. - The mind map of home.

regroupés en croyances, valeurs, connaissances déclaratives (chute, risque de chute, aménagement), connaissances procédurales/prévention du risque de chute, connaissances procédurales systémiques, connaissances procédurales/aménagement du logement, connaissances théoriques. La seconde étape de la théorisation ancrée a consisté à produire une catégorisation de l'identité-logement à partir de l'identification de chacun des deux sentiments constitutifs (SUC et SCT) au sein des codes d'apprentissage. Leur extraction a été suivie de nouveaux regroupements que nous avons nommés catégories A, lesquelles étaient composées de valeurs, croyances et connaissances. Cinq catégories composaient le SUC et 4 catégories, le SCT. Ces catégories ont été testées lors des entretiens 6, 7, 8. L'analyse des verbatim a laissé poindre trois constats (une dissociation difficile entre les deux sentiments ; certaines connaissances procédurales sont des habitudes alors que d'autres sont stratégiques; les personnes ont une perception cyclique du temps présent) appelant à la formulation de trois propositions (le sentiment de continuité temporelle est intégré au sentiment d'unité et de cohérence; le sentiment d'unité et de cohérence peut être stratégique; le sentiment de continuité temporelle est à la fois cyclique et linéaire). Au vu de ces propositions, permettant la mise en lien des deux sentiments, les neuf catégories initiales ont subi une nouvelle catégorisation donnant lieu à six catégories $\mathrm{B}$, composant l'identité logement. La saturation théorique atteinte suite aux entretiens 9 et 10, nous a permis de vérifier les conditions d'existence de chacune des catégories B identifiées (Tab. II), lesquelles étaient suffisantes pour élaborer le modèle expliquant les processus d'apprentissage de l'identité-logement.

\section{Résultats : le modèle de l'identité-logement}

À partir des verbatims recueillis auprès des sujets de l'étude, l'analyse a permis l'émergence de catégories théoriques composant le sentiment de continuité temporelle ( «jonction passé-cycle du présent », «cycle du présent » et «jonction cycle du présent-futur») et spécifiquement le sentiment d'unité et de cohérence («stratégies de défense », «stratégies d'intégration», «stratégies d'adaptation»).

Une première présentation des résultats a consisté à afficher une photographie des catégories finales selon leurs 


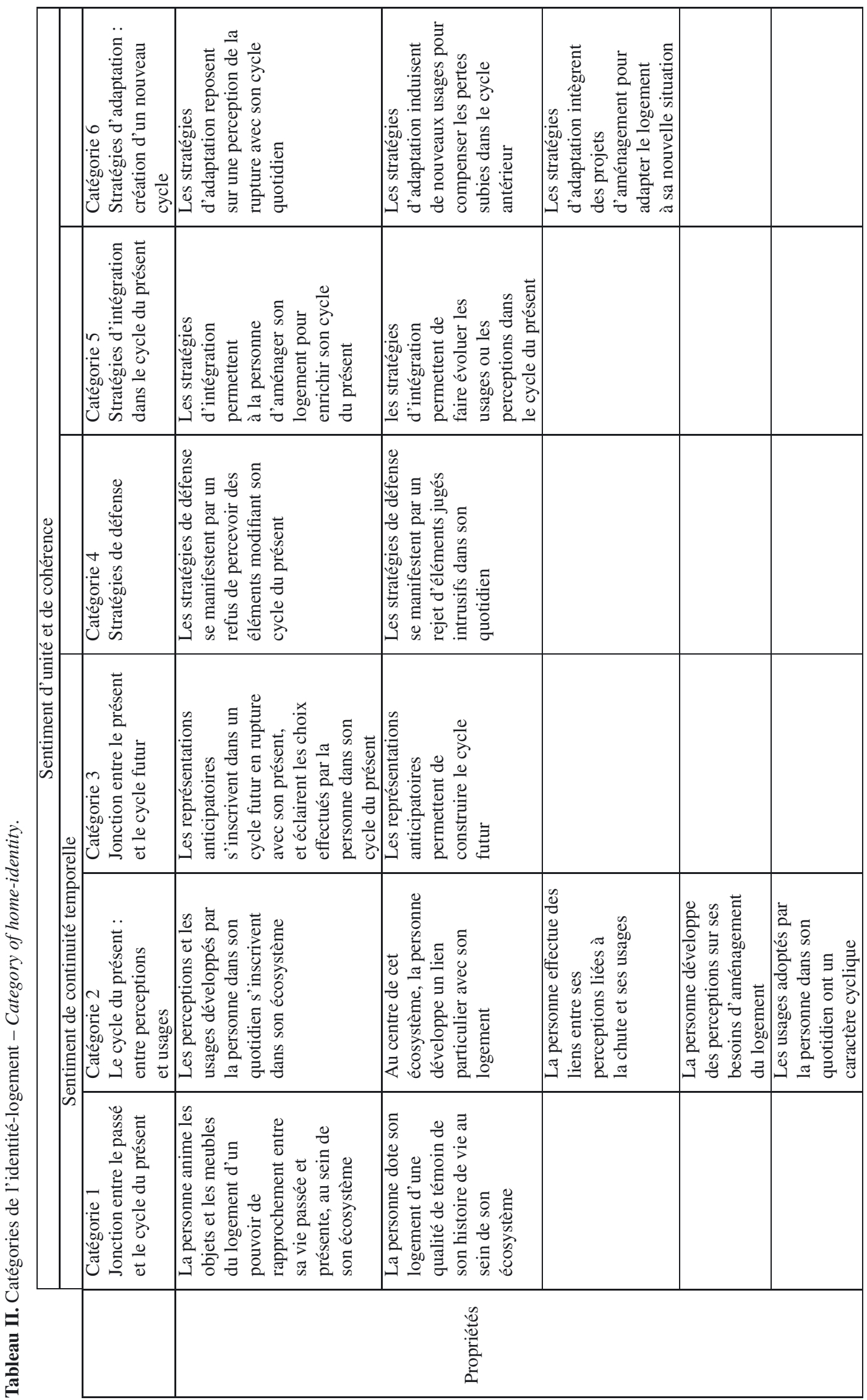


SENTIMENT D'UNITE ET DE COHERENCE = IDENTITE LOGEMENT

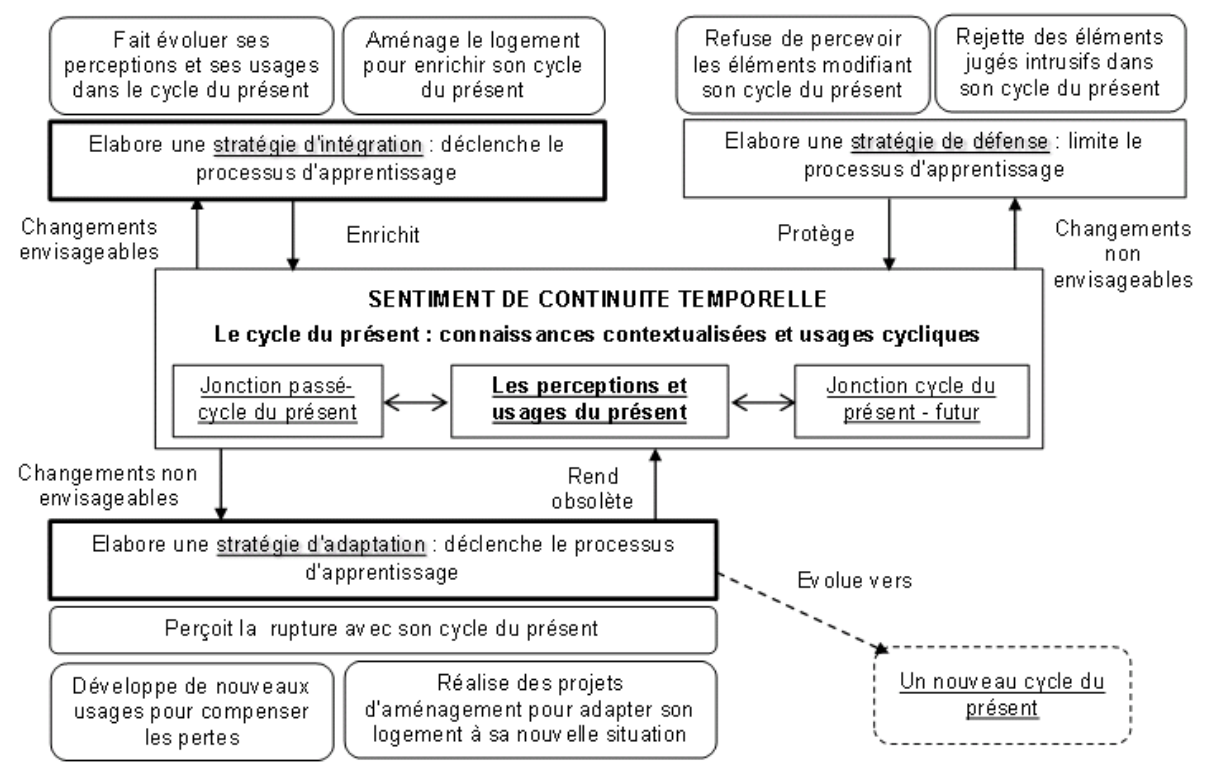

Figure 2. Le modèle de l'identité-logement. - The model of home-identity.

dimensions (étendue de chaque propriété), leurs codes (références au verbatim), leurs conditions d'existence, leurs propriétés et leur définition. L'articulation dynamique de ces catégories a permis de modéliser l'identité-logement (Fig. 2). Le cœur du modèle repose sur le cycle du présent, catégorie constitutive du sentiment de continuité temporelle et catégorie centrale de l'identité-logement. L'individu contextualise ses connaissances et ses usages dans son logement à l'intérieur de ce cycle du présent, sans pour autant les y restreindre puisqu'il les met en lien avec son écosystème, environnement sociospatial plus vaste, selon une temporalité linéaire joignant les éléments de son passé à son présent et à ses projections futures. Le sentiment d'unité et de cohérence contient le sentiment de continuité et, au-delà, offre à l'individu des stratégies pour protéger son identité-logement ou pour la faire évoluer. Selon la stratégie utilisée, le modèle subit des variations différentes. Si les stratégies d'intégration et d'adaptation favorisent l'apprentissage et modifient respectivement la temporalité cyclique et la temporalité linéaire, la stratégie de défense limite l'apprentissage et n'a pas d'impact sur la temporalité de l'individu.

\subsection{Le sentiment de continuité temporelle}

Il se définit par «le cycle du présent : connaissances contextualisées et usages cycliques » regroupant trois catégories : «jonction passé-cycle du présent », «cycle du présent » et «jonction cycle du présent-futur».

Les connaissances liées à l'identité-logement de l'individu sont contextualisées dans le présent au sein du domicile, lequel se situe au centre d'un environnement socio-spatial dont les quatre niveaux influencent les perceptions, les usages et donc les choix effectués par la personne dans son logement. On y retrouve par exemple le poids de l'image négative de la vieillesse imprégnant la société (macrosystème). C : «Et dans la maison vous ne voyez pas d'aménagements autres que ça? M2 : Pour le moment non, sincèrement non. Parce que j'aurai l'impression si on me proposait des trucs, de dire bon ça y est j'ai vraiment pris un coup de vieux là. Oh là.»

Au centre de cet écosystème, l'habitant développe un lien très particulier avec son logement dont la manifestation peut se révéler dans la manière d'investir et d'apprécier les espaces de la maison. Les pièces sont choisies pour des raisons liées au confort, au ressenti mais aussi aux possibilités d'interaction sociale. Une pièce n'est jamais réellement vide. Elle est aussi habitée de souvenirs, baignée de lumière ou au contraire plutôt froide, abrite des sensations mais adopte également les fonctions que lui accorde l'habitant. Ainsi, certains lieux de la maison permettent la rencontre avec le monde extérieur.

M9 : «Ici, là. La cuisine. Parce que souvent j’ai beaucoup de passage. Nous discutons et on coupe le cheveu en 4 pour tout dire. C : - Donc, c'est la cuisine. M9 : - C'est la cuisine, oui. C : - Et pour ces raisons là, parce que vous préférez accueillir? M9 : - Parce que c'est ici que ça se passe. Quand il y a des passages, c'est ici que ça se passe, plutôt qu'à côté. Je déteste recevoir couchée, ça n'a pas de sens. »Une seconde caractéristique du lien entre l'habitant et son logement se définit à travers l'attachement aux meubles composant l'univers quotidien. M2 : «Donc on a récupéré ça. Chaque meuble... Des fois c'est un peu barbant je trouve... de s'accrocher comme ça. Des fois j'ai envie de tout jeter et de dire bon allez hop, c'est que des meubles après tout! F2 : - Oui mais ça ne dure jamais longtemps... M2 :-Bien non, c'est ça le pire [rires]. » La relation entre le corps de l'habitant et son logement apparaît comme une troisième composante de ce lien particulier, déterminant par exemple certains trajets diurnes pour trouver le repos (chambre), ou l'utilisation d'équipements et de mobilier 
pour soulager un corps douloureux ou affaibli (ex : fauteuil). Elle peut être constitutive de perceptions adoptées par l'individu sur des objets du domicile. Ainsi, selon l'avis de certaines personnes interrogées, la mécanique du corps semble s'accoutumer aux objets entreposés dans le logement depuis des décennies. M3 : «Si je veux, je peux l'enlever, hein. C : - Et aujourd'hui, vous pensez que c'est nécessaire? M3 :-Non je ne pense pas parce que, je vous dis, il y a bien 25 ans, 30 ans que j'ai ce tapis là. Le corps est habitué, les jambes sont habituées, encore. Elles sont encore habituées. Ça changera peut-être.» Enfin, ce lien particulier se définit à travers la représentation du logement comme une vitrine se soi-même. L'habitant aménage son logement selon ce qu'il est (M9 : «Venant de Tunisie, j'ai beaucoup de tapis ») mais aussi selon l'image qu'il souhaite renvoyer au monde (F1 : «Non parce que le logement, il était bien. Il était propre et tout alors. M1 :-Alors il dit : pour avoir des douches comme vous, il faut aller loin! C'est les pompiers hein, ils vont tout partout. Ils vont tout partout. F1 : - C'est vrai pour être de 55, regardez le logement comment il est. Il n'y en a pas beaucoup»).

Dans ce contexte, les usages de l'habitant cohabitent avec ses perceptions et s'influencent mutuellement. Ce dernier construit ses connaissances sur le risque de chute au domicile et sur l'aménagement de son logement selon les significations issues de ce lien particulier avec son milieu de vie mais aussi selon des habitudes lui permettant de «fonctionner » au sein de cet environnement familier. Ainsi, le regard de l'habitant sur le risque de chute dans son logement se façonne selon des usages qu'il juge préventifs (A10 : «Tu ne rentres pas dans la cuisine avec le déambulateur? M10 : - Non, non. Avec les chaises, il faut que je pousse les chaises. Non, mais moi, je me tiens grâce à la table et je pousse les chaises »), ou au contraire, jugés risqués $(\mathrm{C}:$ : Les trajets qui vous paraissent les plus dangereux à faire? M4 :-Oh bien c'est le petit couloir puis le voyage pour aller dans la salle d'eau c'est tout. Quand je rentre dans la cuisine, Je n'ai pas de trajet, puis dans la salle à manger je ne bouge pas»). À partir de ses perceptions, l'habitant peut émettre des besoins d'aménagements situés qui diminueraient le risque de chute au domicile (M5 : «La salle de bain, c'est important. La salle de bain, il faut une barre qui mène de la porte jusqu'aux toilettes ») mais aussi des aménagements permettant d'améliorer son quotidien (M9 : «Oui. Le copain qui m'a fait ça [peinture des murs de la cuisine], je voudrais qu'il fasse de l'autre côté, c'est tout. Juste ça, oui. C'est important pour moi. C : - C'est votre priorité ? M9 :-Oui »).

Les perceptions sur le risque de chute ou les aménagements à effectuer ne peuvent donc se détacher des usages rodés dans la vie quotidienne et rythmant les journées de la personne dans son lieu de vie. Ces usages composent les routines de l'habitant et se reproduisent de manière cyclique dans le présent.

Dans notre modèle, la temporalité est cyclique lorsqu'elle accueille les perceptions et les usages routiniers de l'habitant dans sa vie quotidienne. Mais la représentation du temps est aussi linéaire, s'écoulant de façon irréversible et permettant à la personne dans son logement, d'établir une continuité entre ses souvenirs, sa situation présente et ses projections futures. Ainsi le logement, témoin de l'histoire de vie de la personne abrite des souvenirs d'une vie passée ou encore de proches décédés, meublant le présent d'une multitude de significations données aux objets ou simplement aux espaces du domicile. Il arrive parfois que les «choses » composant le lieu de vie soient réparties par les habitants entre les êtres vivants et les morts, rassemblant selon un ordre atemporel, les membres d'une même famille et rétablissant le lien entre passé et présent. M2 : «Mais je ne mets pas les photos des personnes. Vous voyez il n'y a pas mon père, il n'y a pas ma mère. Si, il y a mon mari mais il est caché là haut. [...] F2 : - On n'en $n$ 'a pas besoin de toutes façons, les meubles nous rappellent tous ces gens. M2 : - Parce que, quand on arrive à un certain âge, si on regarde devant il n'y a pas beaucoup de personnes. Quand vous regardez derrière vous, vous dites ouh... Si, il y a une photo qui est là, accrochée au mur là, c'est ma petite fille donc c'est normal qu'elle soit là elle. » Par ailleurs, les représentations du futur renvoient à une vision du possible qui peut influencer les choix présents. Ainsi une projection négative de son futur peut bloquer l'individu dans l'aménagement de son logement alors que l'inverse peut permettre une amélioration du quotidien : M3 : «J'ai eu la folie, j'avais 84 ans, je me suis dit quand même... J'avais des fauteuils qui étaient en skaï blanc qui étaient dégoûtants. C'était sali, c'était incrusté, c'était sale. Et puis j'avais 84 ans, allez, je me suis racheté ces 3 fauteuils. J'ai dit: C'est un monde de racheter ça à 84 ans ! C : - Pourquoi, ils sont beaux! M3 :- Oui, mais justement, à 84 ans! Vous voyez que j'ai foi dans l'avenir! » L'existence de représentations anticipatoires peut être source de projets permettant à l'habitant de préparer son avenir dans le temps présent.

Le sentiment de continuité temporelle est donc composé de connaissances construites sur la base de perceptions et d'usages forgés dans un environnement socio-spatial au sein duquel évolue et interagit l'habitant. Cet environnement comporte également une dimension temporelle à la fois cyclique dans le présent et à la fois linéaire reliant le passé, présent et futur. Le sentiment de continuité temporelle constitue le cœur de l'identité-logement. Il se définit comme l'élément fondamental du concept mais également comme son point faible, ne pouvant supporter de rupture durable.

\subsection{Le sentiment d'unité et de cohérence contient le sentiment de continuité temporelle}

Il donne à l'individu le pouvoir d'user de stratégies. Audelà de la continuité, trois catégories caractérisent le sentiment d'unité et de cohérence :

- La stratégie de défense permet de protéger le sentiment de continuité lorsque la personne pressent que les nouveaux éléments de la situation ne sont pas assimilables en l'état et qu'elle ne perçoit pas d'adaptation possible. Il peut s'agir d'un refus de percevoir des changements qui viendraient modifier son cycle du présent, tel que, par exemple, celui de percevoir des aménagements ou encore le risque de chute à l'intérieur du logement (M2 : «J'avais peur avant dans la salle de bain, avec la baignoire mais comme elle 
n'y est plus, le problème ne se pose plus. Autrement, non je ne me pose pas la question encore. Je me dis j'ai encore le temps. Peut être qu'un jour... non pas dans l'appartement ») ou encore d'un rejet d'éléments jugés intrusifs dans son quotidien (M6 : «Mais l'autre, il m'a emmené un fauteuil [roulant]. En plus, il était large comme ça. Je ne pouvais même pas me rentrer dedans! J'ai dit : Ils sont malades! Non, non, je vous dis c'est presque incroyable. [...] Ben dans le coup, je l'ai mis dans le garage »).

- La stratégie d'intégration permet d'enrichir le cycle du présent, sans le briser, grâce à l'apport de nouveaux éléments dans le quotidien de l'habitant répondant à des besoins existants, tout en modifiant les perceptions et les usages présents. Par exemple, les stratégies d'intégration permettent à la personne d'aménager son logement pour le rendre plus douillet, plus beau ou plus sécurisant vis à vis de la chute ou de faire évoluer ses habitudes pour rendre ses usages plus sûrs. M7 : «Surtout, déjà avec ça (le déambulateur), je ne peux pas descendre les marches. Alors, j'ai arrangé un truc [petit rire] avec ma canne. Heureusement que j'ai un soubassement puis un petit truc comme ça. Alors, j'accroche ma canne là-bas. Alors, je vais avec ça (déambulateur) sur une partie du couloir. Je prends la canne je ... (mime de marcher avec la canne). C : - Vous descendez les marches avec la canne... M7 : - Oh, c'est le parcours du combattant!»

- La stratégie d'adaptation donne à l'individu la possibilité de gérer une rupture de son cycle du présent - lequel deviendra obsolète - en favorisant la construction d'un nouveau cycle. Cette stratégie s'opère dans un premier temps grâce à la perception de cette rupture pouvant se traduire par une séparation avec son environnement social (décès du conjoint, éloignement de la famille, réduction du nombre d'amis, etc.), un arrêt de certaines habitudes (utilisation de la baignoire, sortie de la maison, jardinage, entretien des espaces, etc.), - M9 : «Et ça, c'est la cour. J'attache beaucoup d'importance à la cour parce que c'est fleuri, parce que... Autrefois je m'en occupais beaucoup mais là je ne peux plus. Je n'ai plus de force dans les mains. On ne peut pas être et avoir été. Voilà. »- une dislocation du lien avec son logement (transformation d'un salon en chambre, détérioration de certains espaces, déménagement, etc.). Dans un deuxième temps, la stratégie se manifeste par l'expression de nouveaux usages pour compenser les pertes subies (modification des habitudes, recours à des aides humaines, etc.), - M9 : «C'est plus moi qui m'occupe des plantes. C'est mon fils avec un copain. Je ne peux plus. Avant, c'était moi qui faisais tout. C'est fini! Ça ne me manque pas, franchement. J'adore les plantes mais soulever les pots tout ça... Je ne peux plus. Il faut savoir dire : "Allez, on arrête ». Vous ne pensez pas?» - ou par des projets d'aménagement pour adapter le logement à sa nouvelle situation (installation d'une douche à la place de la baignoire, remplacement de persiennes par des volets électriques, aménagement d'une commode dans la cuisine pour accéder aux dossiers administratifs etc.). M3 : «Alors, j’ai trouvé par Agnès, mais en cherchant sur des catalogues, une petite commode qui est dans le bout de ma cuisine [...]. Toutes mes chemises sont là, toute ma pharmacie, à portée de la main. Tout mon courrier, tous mes papiers, mes impôts, tout ça. J'ai tout à portée de la main, évidemment l'essentiel, c'est d'avoir la possibilité de se mettre tout à portée de la main. Regardez le sel est là le café est là (première étagère du placard). Inaccessible à partir de là. [...] Donc, il s'agit d'aménager aussi en fonction des possibilités qui restent. »

\subsection{Le modèle d'identité-logement selon ces trois stratégies}

Il varie selon ces trois stratégies lesquelles délenchent ou limitent l'apprentissage et agissent sur la temporalité de l'individu. La stratégie de défense en rejettant d'emblée, l'entrée de toute signification nouvelle dans le cycle du présent, ne permet pas l'apport de nouvelles connaissances. Elle constitue un facteur limitant l'apprentissage et n'impacte pas la temporalité de l'individu. Pour protéger l'identité-logement, elle dresse des barrières contre toute perturbation extérieure, œuvre au statut quo du cycle du présent mais ne permet pas d'évolution si la situation de l'individu se modifie.

La stratégie d'intégration permet d'introduire de nouvelles connaissances dans le cycle du présent répondant à des besoins existants et qui s'inscrivent dans la continuité. Elle modifie les perceptions ou les usages courants et permet, à posteriori, une réorganisation des connaissances. M9 : «Il y a un copain qui m'a repeint ça (cuisine). Ça, ça a changé ma façon de voir. Ça fait propre. Il faudra qu'il fasse à côté aussi parce que c'est sale, c'est dégueulasse. Ça, ça a changé ma façon de voir : je me suis dis: Tiens, il va falloir aussi que tu t'y mettes, que tu attendes un petit peu, pour qu'il fasse la suite. »

En aménageant pour réduire le risque de chute, en sécurisant ses trajets ou en améliorant la fonctionnalité du domicile (espaces de circulation, etc.), l'individu peut modifier ses routines, ses habitudes et probablement agir sur sa perception du temps dans le présent. Sa temporalité cyclique peut être modifiée. L'identité-logement s'enrichit en améliorant le cycle du présent actuel.

La stratégie d'adaptation est issue d'une perturbation déstabilisant la vie quotidienne. La continuité subit une rupture. Le changement produit n'est pas envisageable dans le cycle actuel et ne pourra être géré que si l'habitant met en œuvre un apprentissage lui permettant de reconstruire un nouveau cycle. Par exemple, la personne chute et souffre de séquelles. Cette situation impulsera chez l'habitant développant ce type de stratégie, une réorganisation de ses connaissances qui lui donneront la possibilité de percevoir une brèche dans son quotidien. Les habitudes jusqu'alors rodées ne permettent plus à la personne de vivre correctement dans son logement. La personne ne peut plus utiliser la baignoire, ne peut plus sortir de chez elle. Suite à cette perception de l'inefficience du cycle actuel, elle va refonder un nouveau cycle accueillant de nouvelles connaissances pour répondre aux besoins émergeant de cette nouvelle situation (types d'aménagements de la salle de 
bain, services d'aide à domicile, exercices physiques etc.). M6 «Tous les jours je fais mon quart d'heure de culture physique [...] C : - Vous entretenez votre forme... M6 : - Bien, c'est une obligation. Avant, je sortais souvent. Maintenant, je ne sors pas. Bien, je suis obligé de faire des exercices. Comme dans le temps, $j$ 'en faisais. Je suis obligé. Comment faire autrement... » Lorsque l'individu initie une stratégie d'adaptation, il agit très probablement sur sa temporalité linéaire. Une partie de son présent bascule vers le passé et les perceptions de sa vie future son transformées. Avec la construction d'un nouveau cycle du présent, l'identité-logement évolue en modifiant la temporalité linéaire de l'habitant.

\section{Discussion}

\subsection{Le cycle du présent abrite l'agir compétent de l'habitant}

Les résultats nous enseignent que l'identité-logement se structure autour du «cycle du présent » que l'habitant fait fonctionner grâce à ses perceptions et ses routines quotidiennes [32]. Cette identité-logement n'est pas statique puisqu'elle est vouée à être continuellement reconstruite par l'individu, cherchant à maintenir au sein de son logement un « agir compétent» tel que défini par Jonnaert et al. : «L'agir compétent est indissociable de l'intelligence des situations, cette compréhension que les personnes ont des situations et du comment faire pour y être efficaces, soit en y adaptant ce qu'elles sont et leur déjà-là, soit en y construisant de nouvelles ressources » ([33], p. 23). Ainsi, le risque de chute ne relève jamais d'une vision objective puisqu'il est mis en relation avec le regard que l'habitant pose sur son propre agir (usages jugés risqués ou préventifs) dans une situation donnée (activité réalisée, endroit du logement, le moment de la journée, contexte, etc.). Comme le souligne Le Breton la perception du risque ne relève pas d'une vision objective mais d'une projection de sens et de valeurs [34]. Chaque perception construite au sein du logement y compris celle développée sur le risque de chute ou sur les usages, regorge de significations propres au lien développé entre l'habitant et son domicile, au centre de son écosystème. Nous observons par exemple, une aisance des personnes à identifier le risque à l'extérieur du logement plutôt que de le considérer à l'intérieur où l'espace est connu, maîtrisé. Peutêtre pourrions-nous suggérer que le logement représentant un miroir de soi-même et renvoie le reflet de son propre vieillissement. Comment accepter alors de percevoir le risque témoignant de sa fragilité, si l'image des âgés et de leur condition d'existence dans notre société n'est pas acceptable par l'individu? Amyot et Villez [35] notent d'ailleurs que la «reprise en main du domicile» s'effectue par les professionnels et la famille au moment de la fragilisation de la personne âgée, avec un basculement du rapport d'autorité entre les enfants et le(s) parents(s) et une prise de pouvoir par l'entourage, des conditions de vie des anciens. Les auteurs précisent que les soignants auront tendance à hiérarchiser le risque en surévaluant le risque de chute et en sous-évaluant le risque de la perte d'intégrité psychique [35]. Selon nos résultats, nous pouvons donc suggérer que l'habitant construit un agir compétent qui lui est propre, lié à une projection de sens composant l'intelligence des situations. Par cet agir compétent l'habitant développe une maîtrise de son quotidien et maintient son identité-logement grâce à la préservation de sa continuité tant dans le domaine des usages que sur le plan «psychique».

Néanmoins, le sentiment de continuité peut se trouver déstabilisé lorsque cet agir ne permet plus l'efficacité, par exemple, suite à certaines chutes au domicile entraînant des séquelles physiques ou psychologiques. Le sujet aura alors la possibilité de développer des stratégies d'apprentissage issues du sentiment d'unité et de cohérence dans le but de faire évoluer son identité-logement vers un nouvel équilibre. Ce processus correspond à "La construction de nouvelles ressources » évoquées par Jonnaert dans l'élaboration de l'agir compétent [33]. L'habitant développera de nouvelles connaissances sur sa situation laissant émerger de nouveaux besoins (aménagements, aides humaines, aides techniques, etc.).

Au regard de cette analyse, on conçoit que toute intervention préventive de la chute en rapport avec le logement d'une personne âgée «chuteuse » pourrait revêtir une intention éducative. Ne pas prendre en compte cette dimension éducative pourrait s'avérer non seulement improductif (développement de stratégies de défense telles que le rejet des aménagements, le refus de percevoir le risque, etc.) mais parfois dangereux (rupture de l'identité logement, pertes de repères). L'éducation pourrait conduire l'habitant à modifier son identité-logement et à mettre à distance ses stratégies de défense.

L'éducation thérapeutique du patient serait une voie possible pour ce type d'éducation à la prévention de la chute. Lagardère et al. [36] en justifient la pertinence pour plusieurs raisons : tout comme la maladie chronique, la chute n'appelle pas de guérison mais une possibilité d'en réduire les facteurs de risque, d'une part, et se définit comme un processus évolutif, susceptible d'entraîner des complications et donc de réduire la qualité de vie, d'autre part. Selon les auteurs, « $L a$ chute doit [...] être regardée comme une maladie chronique plutôt que comme un accident sans lendemain» ([36], p. 48).

\subsection{Quelle place à l'éducation thérapeutique du patient pour la prévention de la chute?}

L'application de l'éducation thérapeutique du patient à la prévention de la chute est finalement assez récente. Depuis 2014, l'expérimentation PAERPA (Personnes âgées en risque de perte d'autonomie) lancée par la HAS vise à développer une éducation thérapeutique du patient dans quatre domaines : la polymédication, la dépression, la dénutrition et la prévention des chutes. Concernant ce dernier thème, l'ETP est recommandée aux personnes de 75 ans et plus présentant un haut risque de chute ${ }^{1}$, et fait partie intégrante de l'intervention multifactorielle pour plusieurs raisons : «Elle permet d'augmenter

${ }^{1}$ Chutes répétées (au moins 2 chutes depuis 12 mois), ou une chute grave à l'origine d'une fracture, d'une blessure grave, d'une hospitalisation ou d'un temps prolongé au sol (1 h), ou un antécédent de chute dans l'année et un «Time up and Go test » $\geq 14 \mathrm{~s}$. 
les connaissances des facteurs de risque. Elle favorise la prise de conscience par la personne âgée de son risque de chutes. Elle joue un rôle important dans l'acquisition et le maintien de comportements sûrs. Elle augmente l'adhésion du patient âgé aux différentes composantes de l'intervention multifactorielle ». ([17], p. 11). Parmi les compétences d'auto-soins à acquérir par le patient chuteur, référencées par la HAS, figure la compétence «Aménager mon domicile pour limiter les risques de chute» ([37], p. 2). Au regard des résultats de notre étude, l'acquisition de cette compétence nécessiterait un détour préalable par des apprentissages inhérents à l'identité-logement sur la perception des usages (risqués ou préventifs) par l'habitant, ses projections d'aménagement, son utilisation des espaces, son attachement aux meubles, etc.

\subsection{Une possible intervention éducative portant sur l'identité-logement du sujet âgé chuteur dans les programme d'ETP}

Selon l'OMS, l'éducation thérapeutique vise à «aider les patients à acquérir ou maintenir les compétences dont ils ont besoin pour gérer au mieux leur vie avec une maladie chronique " [38]. L'éducation thérapeutique du patient (ETP) s'appuie sur la notion d'apprentissage centré sur le patient dans le but de lui donner tous les moyens cognitifs et techniques d'une cogestion de sa maladie [39]. L'efficacité de l'éducation thérapeutique auprès d'un public âgé n'est plus à démontrer, plusieurs études ayant fait apparaître un développement de compétences et de connaissances chez les seniors atteints de maladies chroniques ayant suivi un programme d'ETP [40-45]. L'apprentissage des sujets âgés chuteurs à l'aménagement de leur logement pour réduire les risques domiciliaires nécessite l'élaboration d'une intervention éducative spécifique, laquelle pourrait s'imbriquer à un programme d'ETP plus vaste traitant de plusieurs facteurs de risque de chute. L'intervention se réfèrerait à l'approche pédagogique de l'éducation thérapeutique du patient de d'Ivernois et Gagnayre [39] et intègrerait des adaptations issues de notre modèle sur l'identité-logement [32].

Elle reposerait sur un cadre pédagogique composé de trois principes fondamentaux :

- une place centrale accordée à la personne âgée en tant que «gestionnaire » de l'aménagement;

- une intervention éducative construite autour du projet d'aménagement de l'habitant;

- une co-construction de la connaissance dans une relation systémique entre l'éducateur, l'habitant, les proches associés au programme.

La spécificité de l'intervention résiderait dans son intégration au milieu écologique du sujet. L'écosystème accueille des ressources humaines, plus ou moins disponibles (proches, professionnels de santé, gardiens d'immeuble, travailleurs sociaux, etc), que la personne choisira ou non d'associer au programme selon les liens de confiance et d'habitudes entretenus avec elles. Cet écosystème peut également porter des valeurs influençant l'adoption de stratégies au cours du programme éducatif, par exemple, des stratégies de défense vis-à-vis de certains objets marqueurs de handicap [14]. Les séances éducatives seraient principalement dispensées au domicile de la personne âgée «chuteuse ». Il ne s'agirait pas de délivrer une connaissance mais de la co-construire sur la base des usages et des perceptions régissant le cycle du présent de l'habitant et que nous avons tenté de mettre en lumière lors de nos travaux. La compétence devient située selon la définition de Jonnaert [46] c'est-à-dire qu'elle est contextualisée dans une situation dont la représentation émane des acteurs. Ainsi, la compétence se co-construirait selon les perceptions émanant du sujet, de ses proches et de l'éducateur. Les influences sociales et culturelles provenant du milieu écologique du sujet doivent être prises en compte par l'éducateur mais avec un objectif de maintenir une centration de la personne âgée dans ce programme et de conserver sa position de gestionnaire de l'aménagement. Ce positionnement permettrait de respecter au maximum ses stratégies de défense (refus par exemple de certains aménagements) tout en s'appuyant sur ses stratégies d'apprentissage (amélioration de l'existant ou construction d'un nouveau cycle quotidien).

De manière logique, les compétences inscrites au programme d'ETP seraient davantage des compétences d'adaptation à la maladie telles que définies par d'Ivernois et Gagnayre [47] destinées à être contextualisées dans des situations de vie des patients. Elles entreraient en jeu dès le diagnostic éducatif, donnant ainsi la possibilité à l'habitant de développer un apprentissage au moment même de la définition de sa situation. Selon Gagnayre «la réflexivité engagée permet au patient au cours même du diagnostic éducatif d'apprendre et de se situer progressivement comme apprenant » [48]. Les besoins éducatifs exprimés par l'apprenant devront provenir à la fois des besoins existants dans son cycle du présent et des besoins émergeants d'une perception de rupture avec ce cycle, si cette dernière existe. Ainsi, il sera nécessaire de permettre à l'habitant de développer une stratégie d'adaptation et donc un apprentissage dès le premier module du diagnostic éducatif. Le second module ne sera destiné qu'aux participants engagés dans ce type de stratégie, les autres apprenants pouvant intégrer directement le troisième module qui conclura le diagnostic éducatif.

Comme le montre la figure 3, Les modules éducatifs pourraient se décomposer en trois parties : le diagnostic éducatif (modules 1-2-3), l'éducation à l'aménagement du logement pour gérer le risque de chute (module 4 ateliers 1 et 2), l'accompagnement et le suivi pendant les travaux d'aménagement.

L'équipe d'éducateurs pourrait être composée de plusieurs professionnels du secteur médico-social dont des psychologues, ergothérapeutes, travailleurs sociaux et référents d'organismes HLM. Chaque module d'éducation comprendrait une compétence à acquérir par l'apprenant, des objectifs pédagogiques à atteindre, une méthode et une évaluation de l'acquisition de la compétence.

Quelques compétences peuvent être proposées à titre d'exemple (Tab. III).

La carte mentale du logement sera utilisée au moment du diagnostic éducatif, lors du module 3 , comme un outil de 
Diagnostic éducatif

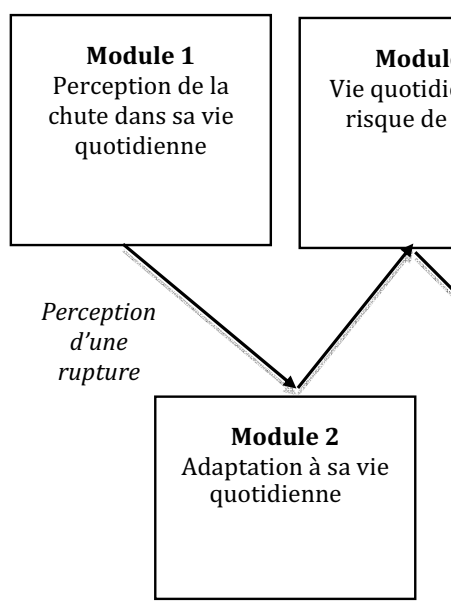

Education à l'aménagement et au risque de chute

Accompagnement

Module 5

Mise en œuvre du projet
Elaboration du projet

d'aménagement
Recherche

aménager son logement et prévenir la chute au domicile

Besoins existants

Stratégie d'intégration

Besoins émergents

Stratégie d'adaptation

Figure 3. Modules éducatifs prenant en compte l'identité-logement. - Educational modules considering home-identity.

Tableau III. Compétences selon les modules sur la réduction des risques domiciliaires. - Skills based modules to reduce home hazards.

\begin{tabular}{|c|c|c|c|c|c|}
\hline Module 1 & Module 2 & Module 3 & Module 4-1 & Module 4-2 & Module 5 \\
\hline $\begin{array}{l}\text { Exprimer } \\
\text { ses } \\
\text { sentiments } \\
\text { relatifs à } \\
\text { la chute }^{1}\end{array}$ & $\begin{array}{l}\text { Etablir des } \\
\text { liens entre la } \\
\text { chute et son } \\
\text { histoire } \\
\text { de vie }\end{array}$ & $\begin{array}{l}\text { Exprimer de } \\
\text { façon située ses } \\
\text { connaissances et } \\
\text { ses besoins dans } \\
\text { son logement }^{3}\end{array}$ & $\begin{array}{l}\text { Analyser des } \\
\text { informations } \\
\text { sur la chute }\end{array}$ & $\begin{array}{l}\text { Formuler un projet } \\
\text { d'aménagement du } \\
\text { logement qui soit } \\
\text { réalisable }{ }^{5} \text { à partir de : } \\
\text { - sa connaissance du } \\
\text { risque de chute en } \\
\text { contexte } \\
\text { - sa connaissance des } \\
\text { équipements et } \\
\text { aménagements (pour } \\
\text { sécuriser l'usage } \\
\text { et alerter) } \\
\text {-des liens développés } \\
\text { avec son logement } \\
\text { (meubles, habitudes, etc.) }\end{array}$ & $\begin{array}{l}\text { Mettre en } \\
\text { œuvre le projet } \\
\text { d'aménagement } \\
\text { du logement }{ }^{6}\end{array}$ \\
\hline
\end{tabular}

${ }^{1}$ Cf. compétence 6 d'adaptation à la maladie « Exprimer ses sentiments relatifs à la maladie et mettre en œuvre des conduites d'ajustement » (d'Ivernois, Gagnayre, 2011, p.S204).

${ }^{2}$ Cf. compétence 7 d'adaptation à la maladie «Etablir des liens entre sa maladie et son histoire de vie » (d'Ivernois, Gagnayre, 2011, p.S204).

${ }^{3}$ Cf. compétence 2 d'adaptation à la maladie « Exprimer ses besoins, solliciter l'aide de son entourage » (Ibid.).

${ }^{4} \mathrm{Cf}$. compétence 4 d'adaptation à la maladie « Analyser les informations reçues sur sa maladie et son traitement » (Ibid.).

${ }^{5} \mathrm{Cf}$. compétence 8 d'adaptation à la maladie « formuler un projet, le mettre en oeuvre » (d'Ivernois, Gagnayre, 2011, p.S204).

${ }^{6} \mathrm{Cf}$. compétence 8 d'adaptation à la maladie «Formuler le projet, le mettre en œuvre » (Ibid.).

représentation de l'identité logement. Elle pourrait ensuite devenir un support à l'évaluation, par exemple au moment de l'élaboration du projet d'aménagement (module 4, atelier 2), pour identifier chez la personne, l'évolution de ses représentations du risque, la modification de ses usages ou une meilleure précision des aménagements à réaliser. En étant accompagné d'un entretien plus approfondi, cet outil pourrait permettre de rendre compte de l'évolution de l'identité-logement de l'habitant suite à l'éducation reçue (module 5).

Cette intervention éducative pourrait exister par ellemême, la réduction des dangers du domicile étant reconnue comme une action efficace en soi [12] mais trouverait davantage de cohérence à intégrer un programme multifactoriel de prévention de la chute. Ainsi, les modules éducatifs pourraient être insérés dans les programmes d'ETP de l'expérimentation PAERPA.

\section{Conclusion}

Notre recherche s'est intéressée à l'existence d'une identité-logement chez les personnes âgées « chuteuses » centrée sur le sentiment de continuité temporelle de l'individu. 
Nous avons tenté de modéliser les processus d'apprentissage et de défense construits par le sujet pour faire évoluer cette identité ou la protéger. Cette modélisation présage de l'importance d'une éducation du sujet âgé chuteur avant toute intervention sur son domicile. En s'adossant aux stratégies d'apprentissage internes à l'identité-logement, elle permettrait de respecter la continuité des usages et des perceptions de l'individu dans son cycle du présent. Cette intervention éducative placerait l'habitant au centre de son projet d'aménagement visant à réduire le risque de chute au domicile. Elle pourrait s'intégrer par la suite à un programme d'ETP multifactoriel de la chute. Cette recherche universitaire donnera lieu à une recherche action ${ }^{2}$ développée au sein d'organismes HLM et qui permettra de formaliser, mettre en œuvre et évaluer cette intervention destinée aux locataires âgés « chuteurs».

\section{Références}

1. Gillespie LD, Robertson MC, Gillespie WJ, Lamb SE, Gate S, Cumming RG, et al. Interventions for preventing falls in older people living in the community. Cochrane Database Syst Rev 2009; 15(2):CD007146.

2. Ricard C, Thélot B. Plusieurs centaines de milliers de chutes chez les personnes âgées chaque année en France. Bull Epidemiol Hebd. 2007; 37-38:322-324.

3. Bourdessol H, Pin S (dir.). Réseau francophone de prévention des traumatismes et de promotion de la sécurité. Référentiel de bonnes pratiques. Prévention des chutes chez les personnes âgées à domicile. Saint-Denis: INPES; 2005, 155 p.

4. Deshpande N, Metter EJ, Lauretani F, Bandinelli S, Guralnik J, Ferucci L. Activity restriction induced by fear of falling and objective and subjective measures of physical function: a prospective cohort study. J Am Geriatr Soc 2008; 56:615-620.

5. Delbaere K, Crombez G, Vanderstraeten G, Willems T, Cambier D. Fear-related avoidance of activities, falls and physical frailty. A prospective community-based cohort study. Age Ageing 2004; 33:368-373.

6. Barry Y, Lasbeur L, Thélot B. Mortalité par accident de la vie courante en France métropolitaine, 2000-2008. Bull Epidemiol Hebd. 2011; 29-30:328-32.

7. Société Française de Documentation et de Recherche en Médecine Générale (SFDRMG), HAS. Prévention des chutes accidentelles chez la personne âgée - Recommandations pour la pratique clinique. Paris: HAS; 2005, 12 p.

8. Société Française de Gériatrie et de Gérontologie (SFGG), HAS. Évaluation et prise en charge des personnes âgées faisant des chutes répétées - Recommandations de bonnes pratiques professionnelles. Paris: HAS; 2009, 19 p.

9. Chevalier P. Société Scientifique de Médecine Générale. Prévention des chutes chez les personnes âgées. Synthèse de la mise à jour (mars 2008). Recommandations de Bonne Pratique. Revue de la Médecine Générale 2008; 254 (supp 1): 1-31.

2 Obtention d'un appel d'offre en avril 2014, lancé par la Fédération des ESH (Entreprises Sociales pour l'Habitat). La recherche action sera menée auprès de 20 locataires d'ESH du Groupe Arcade dans le cadre d'un partenariat entre CPH, l'ALFI et le LEPS EA3412.
10. American Geriatrics Society, British Geriatrics Society. Summary of the updated American Geriatrics Society/British Geriatrics Society clinical practice guideline for prevention of falls in older persons. J Am Geriatr Soc 2011; 59:148-157.

11. U.S. Preventive Services Task Force, Moyer VA. Prevention of falls in community-dwelling older adults: U.S. Preventive Services Task Force recommendation statement. Ann Intern Med 2012; 157:197-204.

12. Gillespie LD, Robertson MC, Gillespie WJ, Sherrington C, Gates $\mathrm{S}$, Clemson LM, et al. Interventions for preventing falls in older people living in the community. Cochrane Database Syst Rev 2012; 9:CD007146.

13. Fondation Abbé Pierre. Rapport annuel 2009. L'état du mallogement en France. Liévin : L'artésienne, 2009 : 319 p.

14. Clément S, Rolland C, Thoer-Fabre C. Usages, Normes, autonomie : analyse critique de la bibliographie concernant le vieillissement de la population. La Défense: Ed. Recherche du PUCA; 2007.

15. Morestin F, Cauvin S. Personnes âgées et adaptation du logement. Présentation, résultats et prospectives du projet de l'étude SADEER. Gérontologie et Société 2011; 136:141-155.

16. Trévidy F, D'Ivernois JF, Gagnayre R, Mourad JJ. Identitélogement et gestion du risque de chute chez les personnes âgées. Le sujet dans la Cité 2011; 2:109-120.

17. HAS. Cahier des charges pour la mise en oeuvre de l'éducation thérapeutique du patient (ETP) dans le cadre de l'expérimentation PAERPA (personnes âgées en risque de perte d'autonomie) Note méthodologique et de synthèse documentaire - Efficacité de l'éducation thérapeutique du patient (ETP) dans le cadre des programmes PAERPA. Paris: HAS; 2014, 67 p.

18. Djaoui E. Le domicile comme espace psychique. Documents Cleirppa 2006; 21:8-11.

19. Ennuyer B. Quelles marges de choix au quotidien quand on a choisi de rester dans son domicile. Gérontologie et Société 2009; 4(131):63-79.

20. Bachelard G. La poétique de l'espace. Paris: Presses Universitaires de France; 1957.

21. Yates FA. L'art de la mémoire. $2^{\mathrm{e}}$ éd. Paris : Gallimard; 1987.

22. Leduc F, Delcourt JB. Aider Soigner Accompagner les personnes à domicile : un éclairage sur l'intime. Gérontologie et Société 2007; 122:145-50.

23. Dreyer P. Limiter les conséquences de la vieillesse et de la dépendance : Agir sur l'habitat et l'environnement. Gérontologie et Société 2008; 125:167-84.

24. Le Borgne-Uguen F, Pennec S. L'adaptation de l'habitat chez les personnes de plus de 60 ans souffrant de maladies et de handicaps et vivant à domicile. Usages et interactions entre les personnes, les proches et les professionnels à travers les objets, les techniques et les aménagements [Contrat de recherche MiRe et CNAV : Atelier de Recherche Sociologique]. Brest : Université de Bretagne Occidentale, 2000: 212 p.

25. Piaget J. La naissance de l'intelligence chez l'enfant. 9e éd. Neuchâtel: Delachaux et Niestlé; 1977.

26. Moser G. Psychologie environnementale. Les relations hommeenvironnement. Bruxelles: De Boeck; 2009.

27. Proshansky HM. The city and self-identity. Environment and Behaviour. 1978; 10:147-69.

28. Bronfenbrenner U. The ecology of human development: experiments by nature and design. Cambridge (Etats-Unis): Harvard University Press; 1979. 
29. Mucchielli A. L'identité. $7^{e}$ édition. Paris: Presses Universitaires de France; 2009.

30. Paillé P. L'analyse par théorisation ancrée. Cahiers de recherche sociologique 1994; 23:147-181.

31. Glaser BG, Strauss AL. The Discovery of Grounded Theory: Strategies for Qualitative Research. Chicago: Aldine Publishing Company; 1967.

32. Trévidy F., Ivernois (d') JF, Mourad, JJ. Vieillir en Santé à son domicile : apprendre pour mieux gérer le risque d'isolement social. Une modélisation de l'Identité-logement au service de la prévention de la chute. Thèse de doctorat en sciences de l'éducation. Université Paris 13; 2013: 581 p.

33. Jonnaert P, Barrette J, Masciotra D, Yaya M. La compétence comme organisateur des programmes de formation revisitée, ou la nécessité de passer de ce concept à celui de «l'agir compétent ». Observatoire des réformes en éducation; 2006 [Consulté le 03 février 2013]. Disponible à l'URL: http://www.ibe.unesco. org/French/cops/Competencies/ORE_French.pdf.

34. Le Breton D. Sociologie du risque. Paris: Presses Universitaires de France; 1995.

35. Amyot JJ, Villez A. Risque, responsabilité, éthique dans les pratiques gérontologiques. Paris: Dunod; 2001.

36. Lagardère P, Pardessus V, Beghin V, Sepieter C, Petit V, Puisieux F. Introduire une démarche éducative dans la prise en soin du sujet âgé chuteur. La revue de gériatrie 2013; 38:47-57.

37. Haute Autorité de Santé. Cadre référentiel ETP Paerpa Prévention des chutes. Paris : HAS, Septembre 2014 : 6 p.

38. Organisation Mondiale de la Santé. Éducation thérapeutique du patient : programme de formation continue pour les professionnels de soins dans le domaine de la prévention des maladies chroniques, recommandations d'un groupe de travail de l'OMS. Copenhague: Bureau régional pour l'Europe; 1998, $57 \mathrm{p}$.
39. Ivernois (d') JF, Gagnayre R. Apprendre à éduquer le patient. $4^{\mathrm{e}}$ éd. Paris : Maloine; 2013.

40. Crozet C, Van Bockstael V, Devos J, D'Ivernois JF. Évaluation d'un programme national en France d'éducation thérapeutique pour des patients du régime agricole atteints de maladies cardio-vasculaires. Educ Ther Patient/Ther Patient Educ 2009; 1(1):33-38.

41. Schiel R, Braun A, Muller R, Helbich C, Siefke S, Franke I et al. A structured treatment and educational program for patients with type 2 diabetes mellitus, insulin therapy and impaired cognitive function (DikoL). Medizinische Klinik 2004; 99(6):285-292.

42. Zeyfang A. Structured educational programs for geriatric patients with diabetes mellitus. MMW Fortschr Med 2005; 147(26):43,45-46.

43. Mosnier-Pudar H. L'éducation du patient diabétique âgé. La revue de gériatrie 2011/12; 36(10):715-718.

44. Bonsack S, Spirig R, Baldegger E, Wettstein A. Results of the Qualitative Evaluation of all-fit-a group education and training program for elderly people with chronic illness. Pflege 2006; 19:244-250.

45. D'Ivernois JF, Crozet C. Pour une nouvelle approche des soins. Soins 2003; 675:34-36.

46. Jonnaert P. Compétences et socioconstructivisme. Un cadre théorique. $2^{\mathrm{e}}$ éd. Bruxelles: De Boeck; 2009.

47. Ivernois (d') JF, Gagnayre R, les membres du groupe de travail de l'IPCEM. Compétences d'adaptation à la maladie du patient : une proposition. Educ Ther Patient/Ther Patient Educ 2011; 3(2):S201-S205.

48. Gagnayre R. Un modèle pédagogique comme modèle d'intervention pour aider les patients diabétiques à acquérir des compétences d'autosoins et d'adaptation à leur maladie. In: Ivernois (d') JF (dir.), Pratiques de l'éducation thérapeutique du patient dans le diabète. Paris: Maloine; 2012: p. 111-126. 